vacation work supports the student population in many other countries where more school leavers than in Britain go on to university. These are only some solutions based on practice in other countries. What is certain is that our universities will get little public sympathy in the current economic climate if they simply hold out their begging bowls. As we said in January, ${ }^{5}$ the multiplicity of the academic talking shops makes procrastination easier than making decisions-but action is needed if disaster is to be averted.

This five-year crisis in academic medicine should be seen as a warning by the staff of the NHS. They, too, can see clear signs of ever-worsening financial problems. The Government's policy (on this and other issues) seems to depend on a conviction that an opinion repeated often enough becomes a fact. More money has to be found for health care in Britainbecause on objective measures the population of our country is not getting adequate medical treatment. How that money is found-from taxation, from health insurance, or from lotteries - is a political issue. The Government will be forced to face that issue only by public pressure.

The time may, indeed, have come for doctors to put the good of the community ahead of the needs of their individual patients. Data just published ${ }^{1}$ show that whereas West Germany, Italy, and France treat 70 patients per million population in the age range 55 to 64 for end-stage renal failure the NHS can manage only 22 per million; and in the over- 65 s our neighbours treat 50 per million against 3.5 by the NHS. When a clinician knows that his own patient cannot have some treatment or investigation which is available to most patients in Europe or the United States, his instinctive reaction is to tell the patient or the relatives that nothing more can be done. That is the wrong response. If he speaks out and says what the NHS ought to be providing but is failing to provide, his patient may be distressed. But, distressing as it may be for a patient and her family to know that treatment is possible but is not available, the alternative-pretending there is no suitable treatment-is surely a greater offence against medical morality.

\footnotetext{
1 South-east Thames and South-west Thames Regional Health Authorities. Renal services. Croydon and London: South-east Thames Regional
Health Authority and South-west Thames Regional Health Authority, 1982.

2 Steiner RE. New imaging techniques: their relation to conventional radiology. Br Med f $1982 ; \mathbf{2 8 4}: 1590-2$.

${ }^{3}$ Smith R. No apples for the teachers. Br Med $\mathcal{F} 1982 ; 284: 1965-6$.

4 University of London. London medical education-a new framework. Report of a Working Party on Medical and Dental Teaching Resources, chairman Lord Flowers. London: University of London, 1980.

5 Anonymous. Cuts and excellence. $\mathrm{Br} M e d \mathcal{F}$ 1982;284:294.
}

\section{Cataract surgery}

Improvements of surgical technique, instrumentation, and materials have reduced the frequency of many of the complications of cataract surgery, but ophthalmic surgeons still differ in their choice of operative technique. What have been the changes in recent years?

The microscope has given the surgeon a much more detailed view of the operative field, providing him with greater visual feedback and allowing, therefore, more manual control. The fine needles now used in cataract surgery hardly need tissue fixation in order to pass through the ocular structures. Accurate wound closure with smooth non-irritating materials makes the eye more comfortable in the early postoperative course and also much safer. Patients may be mobilised more quickly, return home earlier, and resume activities after a shorter convalescence.

A cataract can be removed with its capsule (intracapsular removal) or without its capsule (extracapsular removal). ${ }^{1}$ Both operations remove the fog, dazzle, distortion, and other discomforts suffered by the patient as cataract advances. Both have their advantages and disadvantages, and surgeons have their preference in particular cases. In both methods there have been developments which have made for safer surgery.

Intracapsular extraction exposes the face of the vitreous to damage, and this may lead to postoperative problems. The enzyme alpha-chymotrypsin, which acts on the suspensory ligament of the lens, makes the operation safer and easier. The lens itself can be held by capsule forceps, by suction, or by freezing with a cold probe at $-40^{\circ} \mathrm{C}$. The latter method is by far the safest, since it provides a grip not only on the lens capsule but also through it into the substance of the lens itself.

During recent years there has been a rebirth of enthusiasm for extracapsular extraction as refined by the use of new instruments. In the older method the extraction of the lens cortex was incomplete, causing irritation of the eye, an iritis, and sometimes a severe immune response. Thickening of the lens remnants might obstruct the pupil, and results were not as good as with the intracapsular method. This has now changed. Extracapsular extraction may now be performed through a much smaller incision, reducing the risk of some complications. The nucleus of the lens may be removed with a microcutter or by ultrasonic emulsification. The microscope allows the surgeon to see all the lens cortex in detail, pick it up piece by piece, and peel back to the equator of the lens in a wedge and then from the surface of the posterior capsule.

Several claims and counterclaims are being made about the merits of intracapsular and extracapsular extraction, ${ }^{2}$ but the final result and the total incidence of postoperative complications are much the same with both methods. Their expectation of success is high and the risk of serious complication low.

Postoperatively, spectacles give greatly improved visual acuity, but many problems remain, to which some patients can never adapt. The visual image is enlarged, the visual field limited, and the prismatic and peripheral aberrational effects require that the head is moved rather than the eyes. Judgment of distance is impaired, leading to clumsiness in performing simple tasks, and there is no prospect of binocular vision unless both eyes are in the same condition. Contact lenses overcome many of these problems, but most patients with cataracts are elderly and are slow to adapt and learn. Contact lenses designed for prolonged wear have helped some patients but have not proved fully satisfactory.

The ideal position for any optical correction is as close to the normal site of the lens as possible, and the insertion of an intraocular lens immediately after cataract extraction is now possible with an acceptable level of safety. It demands high surgical skill and a careful choice of method. Temporary loss of the anterior chamber or a small peripheral adhesion of the iris would probably not threaten the end result in orthodox cataract surgery, but when an intraocular lens has been inserted these complications are more important and may lead to irreversible damage to the corneal endothelium. Even momen tary contact can destroy thousands of endothelial cells on the posterior corneal surface.

Nevertheless, if patients are suitably selected and proper precautions are observed the incidence of complications with intraocular lens surgery should differ little from those of orthodox 
cataract surgery in the same hands. Rehabilitation is much easier than with other methods. The postoperative vision and orientation seem natural, and the patient can often see well without an additional optical correction. With this technique cataract surgery can be offered to patients with unilateral lens opacities which used to be left untreated when the fellow eye remained unaffected.

Provided, therefore, that preoperative assessment excludes the presence of other conditions affecting the eye and any general disease which would have an adverse influence, the prospects for cataract surgery are good. In uncomplicated cases over $95 \%$ of patients may expect to achieve a final corrected vision of $6 / 12$ or better.

M J ROPER-HALL

Consultant Ophthalmologist,

Birmingham B15 3HE

1 Roper-Hall MJ, ed. Stallard's eye surgery. 6th ed. Bristol: Wright, 1980.

${ }^{2}$ Ruben M, Choyce DP, Roper-Hall MJ, et al. Symposium on the correction of aphakia. Trans Ophthalmol Soc UK 1981;101:48-86.

\section{Do alcoholics recover?}

Doctors weary of exhortations to identify alcoholics, and many resent the suggestion that they should be treating them, too. Do not alcoholics inevitably slide downhill, perhaps with brief plateaux, with few if any recovering? This stereotype might, however, be based only on a few recalcitrant and unrepresentative bad pennies. Doctors seldom meet recovered alcoholics in their clinics or surgeries. What are the prospects for recovery? And how much difference is made by medical intervention?

A series of surveys in the United States, with follow-ups after two to three years, have shown that people move into and out of problem drinking. ${ }^{12}$ Two-thirds have improved by the second interview and half are free of problems. Even if follow-up is complete (which is only sometimes the case ${ }^{1}$ ) such surveys may be criticised. Possibly they may not detect "real" alcoholics in the first place: they may miss homeless individuals and people who are never in when the interviewer calls. Perhaps, moreover, "real" alcoholics go undetected because they lie.

Neither these two criticisms nor the pitfall of loss to follow-up applied to two recently reported longer-term studies, both of defined populations. The Lundby cohort consisted of all adults living in a Swedish town on 1 July 1947. In 1957 all were reinterviewed by a psychiatrist, and 96 of the 1312 men were classified as alcoholics. They were interviewed again 15 years later, and information from official sources, families, and neighbours collated. ${ }^{3}$ Of the 96 alcoholics, 29 had recovered, 42 were unchanged or worse, and 25 had died (though only four of them died below the age of 65).

Vaillant and Milofsky have used data on inner-city teenagers originally studied in the 1940 s and reinterviewed at ages 25 , 31 , and $47 .^{4}$ Arrest and clinic records were obtained. The criteria for alcohol abuse at some point in their lives had been fulfilled in 110 men. By the age of 47 some 35 of these 110 men continued to abuse alcohol and could be termed progressive alcoholics. Nevertheless, 38 were abstinent and 21 of them had achieved at least three years of abstinence (average 10 years); 26 were still drinking, but without any problems in the preceding 12 months, and 18 of these had achieved two or more years of asymptomatic drinking (average 10 years).

Recovery rates reported from clinics are similar or slightly $\frac{3}{2}$ worse, with $20-40 \%$ of patients doing well and another third 은 somewhat improved. ${ }^{5-7}$ Though on average they are probably $\overparen{\Omega}$ not "severer" alcoholics than those found in community $\overline{\bar{J}}$ studies, clinic patients might include a higher proportion of $D_{0}$ socially dislocated people. Perhaps, too, clinics may attract $\$$ patients with tendencies to depression or passivity, which $\stackrel{0}{0}$ might augur for a bad prognosis. Moreover, people who seek help or are coerced to attend a clinic are likely to be those who have already failed to "cure" themselves or who are reluctant 0 to try.

Treatment may actually make some alcoholics worse, by $\frac{\bar{\omega}}{\widehat{\phi}}$ sheltering them from the consequences of their drinking or $\varnothing$ fostering passivity. The gauntlet has been thrown down ${ }^{8}$ : क the onus of proof is now on therapists who try to provide $\overrightarrow{0}$ more than just succour to show that they are necessary agents $\vec{\overrightarrow{ }}$ in the recovery of certain of their patients and to show which $\stackrel{\omega}{\sigma}$ these are.

Recovery of alcoholics detected in community studies is sometimes but not often attributable to treatment. ${ }^{134}$ Both ơ in clinics and in surveys the best predictor is stability in one's job and marriage..$^{359}$ While important in the Lundby study, $\mathbb{W}^{-}$ severity, in terms of degree of dependence and number of $i$ symptoms, did not emerge as an important factor in Vaillant's 응 data. The link between good outcome and social stability is $\omega$ sometimes thought to be attributable to personality: secure, conscientious people who choose their job and spouse wisely also tackle their drinking problem seriously. Knupfer found that good outcome was commoner in those who reported a ? stable childhood, but this was a retrospective assessment. ${ }^{1}$ Vaillant, who had data uncontaminated by the patient's memory, did not find a link between childhood disturbance and poor outcome. Sociopathy did not predict outcome in either of these community-based studies.

All these follow-up studies (and many others ${ }^{10}$ ) have found alcoholics who have gone on to live healthy stable lives yet have continued to drink. Those who achieve this result are usually younger and have experienced fewer symptoms than average. Probably abstinence is seriously attempted only by those whose distress is so severe that a radical solution seems commensurate; or individuals who are conscientious, can organise their lives, and have self-control may not let $\dot{\delta}$ their drinking get too bad before they do something about it. ₹ They then successfully apply those traits to changing their $\frac{\text { o }}{2}$ pattern of living in such a way that their drinking is never to $N$ excess. But it is also possible that a physiological factor $\rightarrow$ contributes-less severely dependent drinkers might be less prone to reinstate the vicious circle of withdrawal symptoms and relief drinking which leads to relapse in those who are $N$ severely dependent.

The route by which individuals "spontaneously" recover is obviously of interest to the beleaguered clinician. Change of job; a new relationship; criticism from family or friends; a $\stackrel{?}{+}$ disturbing illness-these are some of the more commonly mentioned factors. ${ }^{1112}$ Some describe finding a substitute: tranquillisers, eating, meditation, work, hobbies. Of Vaillant's securely abstinent men, a third had used Alcoholics Anonymous. Clearly, however, many individuals recover without incorporating into their view of themselves that "I am an alcoholic."112 Fresh hope and new self-esteem were important elements in the recovery of the men in Vaillant's study. Our understanding of alcoholism will be restricted if 